\title{
Ipilimumab-induced hypophysitis: MR findings
}

\author{
Franz A. Fellner*1,2, Rainer Wunn ${ }^{1}$, Christine M. Fellner ${ }^{1}$ and Mario Scala ${ }^{1}$ \\ ${ }^{1}$ Central Radiology Institute, Kepler University Hospital, Medical Faculty of the Johannes Kepler University, Linz, Austria \\ ${ }^{2}$ Medical Faculty of the Friedrich-Alexander-University of Erlangen-Nürnberg, Germany
}

A Clark Level IV melanoma of the left thoracic wall metastasizing in axillary lymph nodes, lungs, and brain was treated with Ipilimumab. 14 days after an Ipilimumab cycle the patient came with reduced general condition, severe headache, and polyuria. Laboratory examinations revealed low serum levels of fT4, fT3, TSH, testosterone, and DHEAS.

An MR examination of the brain and pituitary gland revealed diffuse enlargement of the hypophysis involving the pituitary stalk (Figure 1c,d). A previous MR scan of the brain, done 4 months before, showed at that time a regular-sized and shaped pituitary gland and pituitary stalk (Figure 1a,b). Therefore, the diagnose was acute secondary hypophysitis. After that, the patient was treated with dexamethasone. An MR control 17 days later demonstrated complete remission with normalization of the size, shape, and position of the pituitary gland and stalk (Figure 1e,f). Also laboratory parameters were normalized.

Ipilimumab (MDX-010), a human monoclonal antibody, is used for treatment of metastasizing resp. unresectable melanoma[1]. Ipilimumab augments the immune response against tumors by overcoming the suppressive effects of cytotoxic T-lymphocyte antigen 4 expression [2]. However, this may lead to immune-related side effects,

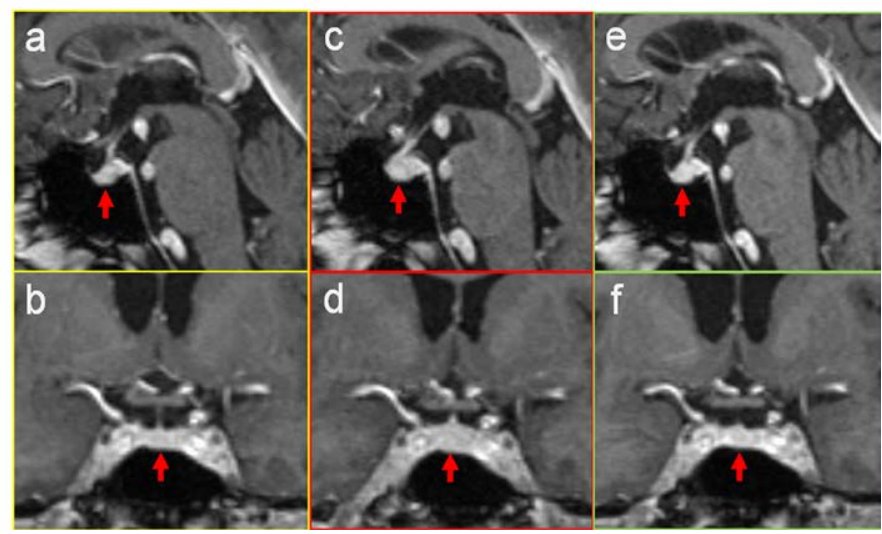

Figure 1. Contrast-enhanced sagittal $(\mathrm{a}, \mathrm{c}, \mathrm{e})$ and coronal $(\mathrm{b}, \mathrm{d}, \mathrm{f})$ images of the pituitary gland (arrows) before (a,b), during (c,d) and after successful treatment (e,f) of ipilimumabinduced hypophysitis. In the stage of acute secondary inflammation there is diffuse enlargement of the pituitary gland and slight thickening of the pituitary stalk (c,d). After therapy the gland is re-sized to normal volume $(e, f)$.

Copyright: (C)2017 Fellner FA. This is an open-access article distributed under the terms of the Creative Commons Attribution License, which permits unrestricted use, distribution, and reproduction in any medium, provided the original author and source are credited. such as secondary inflammatory lesions (dermatititis, arthritis, colitis, uveitis, or hypophysitis).

The term hypophysitis“ includes two entities: primary and secondary hypophysitis. „Primary hypophysitis“ is a lesion of unknown etiology and histologically described as a lymphocytic, granulomatous, or xanthogranulomatous inflammation of the pituitary gland[3]. "Secondary hypophysitis“, on the other hand, is a direct inflammatory or infectious lesion or an involvement of the pituitary gland due to adjacent lesions, such as tumors (adenoma, craniopharyngeoma, germinoma) or rupture of a Rathke cleft cyst $[4,5]$. Immunotherapy with T-cell responses stimulating antibodies is a promising new treatment option for cancer patients, but may have relevant adverse effects, such as hypophysitis. Clinical symptoms and pathologic changes of laboratory values concerning sodium and pituitary hormons in patients treated with Ipilimumab should alert one to think of hypophysitis. Early MR for detection and consequently immediate start of therapy are important to avoid late complications of hypophysitis, such as insufficiency of the adrenal glands.

\section{References}

1. Hodi FS, O’Day SJ, McDermott DF, Weber RW, Sosman JA, et al. (2010) Improved survival with ipilimumab in patients with metastatic melanoma. $N$ Engl J Med 363: 711-723.[Crossref]

2. Keilholz U (2008) CTLA-4: Negative regulator of the immune response and a target for cancer therapy. J Immunther 31: 431-439.[Crossref]

3. Cheung CC, Ezzat S, Smyth HS, Asa SL (2001) The spectrum and significance of primary hypophysitis. J Clin Endocrinol Metab 86: 1048-1053.[Crossref]

4. Sautner D, Saeger W, Lüdecke DK, Jansen V, Puchner MJ (1995) Hypophysitis in surgical and autoptical specimens. Acta Neuropathol 90: 637-644.[Crossref]

5. Dillard T, Yedinak CG, Alumkal J, Fleseriu M (2010) Anti-CTLA-4 antibody therapy associated autoimmune hypophysitis: serious immune related adverse events across a spectrum of cancer subtypes. Pituitary 13: 29-38.[Crossref]
Correspondence to: Franz A. Fellner,Central Radiology Institute, KeplerUniversity Hospital, Medical Faculty of the Johannes Kepler University, Linz,Austria, Medical Faculty of the Friedrich-Alexander-University of ErlangenNürnberg,Germany, E-mail: franz.fellner@kepleruniklinikum.at

Received: May 05, 2017; Accepted: May 18, 2017; Published: May 22, 2017 
